\title{
Barbier allylation of aldehydes and ketones with aluminium and catalytic indium metal: an economical alternative
}

\author{
Marcelo D. Preite,* Hugo A. Jorquera-Geroldi, and Andrés Pérez-Carvajal \\ Departamento de Química Orgánica, Facultad de Química, Pontificia Universidad Católica de \\ Chile, Campus San Joaquín, Vicuña Mackenna 4860, Santiago, Chile \\ E-mail: mpreite@uc.cl
}

Dedicated to Professors Manuel González Sierra, Julio C. Podestá, Rita Hoyos de Rossi, and Oscar S. Giordano, in recognition of their achievements in organic chemistry

\begin{abstract}
An economical preparative protocol for the Barbier allylation of aldehydes and ketones in DMF, using aluminium foil in the presence of a catalytic amount of indium metal, is reported. All yields obtained are in general similar but slightly lower than those reported for the stoichiometric indium allylation. Aluminium alone failed to give rise to any detectable product.
\end{abstract}

Keywords: Barbier reaction, allylation, aluminium allylation, indium-catalyzed allylation

\section{Introduction}

The Barbier allylation of carbonyl compounds finds many applications in organic synthesis. ${ }^{1}$ The main difference between a Barbier and a Grignard reaction is that the former is a one-pot process, with the metal, the alkylating species and the carbonyl present from the beginning of the reaction, in contrast with the later, a two-step procedure that requires the formation of an alkyl metal in the first step, followed by its reaction with a carbonyl, as a second, separate step. Particularly useful, and subsequently extensively studied, is allylation, due to the high reactivity of allyl halides towards many low valent metals. Among them, $\mathrm{Li},{ }^{2} \mathrm{Mg},{ }^{3} \mathrm{Mn},{ }^{4} \mathrm{Zn},{ }^{5} \mathrm{Sn},{ }^{6} \mathrm{~Pb},{ }^{7} \mathrm{Bi},{ }^{8}$ $\mathrm{Ce},{ }^{9}$ and many others, ${ }^{10}$ have been used in Barbier allylations, and the characteristic features of each metal has been well documented.

One of the most remarkable is the indium-mediated Barbier allylation of aldehydes and ketones, now a very well established method for $\mathrm{C}-\mathrm{C}$ formation, after two decades of constant developments since its discovery. ${ }^{11}$ Indium powder is particularly useful and reactive for allylations, which can be performed in either organic or aqueous media, with an allyl halide (usually a bromide or an iodide). ${ }^{12 a}$ Recently we found that the granular form of In metal can 
also be used as a cheaper alternative to powder, which is very convenient in the preparative scale; the price of In powder can be ten fold that of the granular form. ${ }^{12 b}$ Owing to the many uses of indium particularly in technology such as in LCD and television screens, semiconductors, solar technology, eco-friendly solder, etc., their has been over the last decade an increased demand and subsequently greatly increased costs. ${ }^{13 a}$

Here, we report further progress in more economical Barbier allylation methods, using catalytic amounts of granular indium metal, together with aluminium foil as the stoichiometric metal.

\section{Results and Discussion}

In vigorously stirred $\mathrm{N}, \mathrm{N}$-dimethylformamide, the use of a catalytic amount of In metal together with another metal able to reduce back the oxidized indium species formed at $40-50{ }^{\circ} \mathrm{C},{ }^{12 \mathrm{~b}}$ gives rise to efficient Barbier addition reactions of allyl bromide on aldehydes or ketones. ${ }^{13}$ There were several previous reports confirming that the electroreductive regeneration of low-valent indium could be achieved substituting most of the In metal by other metals such as powdered aluminium, zinc or manganese.$^{14}$ Triallylaluminium, however, which can add to some carbonyl compounds and related imines, ${ }^{15}$ unfortunately required the use of highly toxic mercury salts for the activation of the metal surface, so creating a serious problem with their handling, storage, and waste disposal.

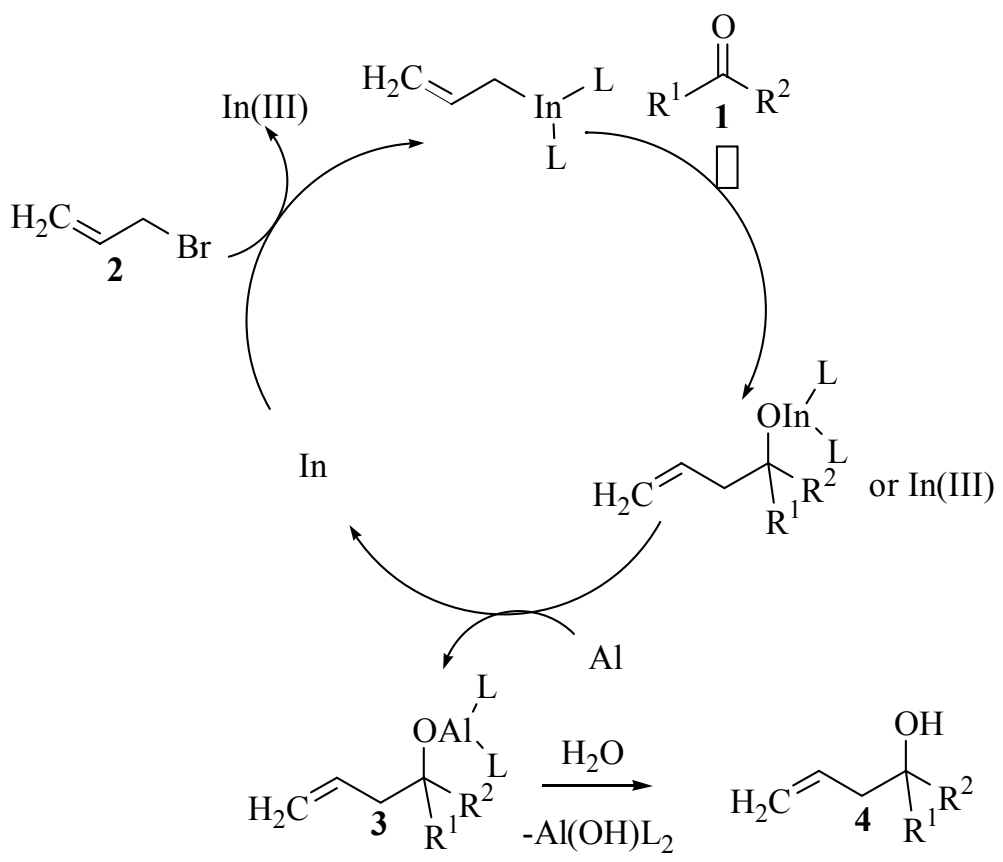

Scheme 1. Catalytic reaction cycle. 
In our hands, the best results were obtained when Al was used as stoichiometric metal, together with a catalytic amount of In (Scheme 1). At first, we tested aluminium powder, but after some tests we switched to a cheaper and safer source of metal: household aluminium foil, which actually worked better than the powdered metal. ${ }^{16}$

When an aldehyde or ketone $\mathbf{1}$ was dissolved in an appropriate solvent (we used DMF, but other polar organic solvents, or even water, can be used), together with allyl bromide (2, $\mathrm{X}=\mathrm{Br})$, and vigorously stirred in the presence of a catalytic amount of granular In metal (0.01 to 0.1 equiv), $\mathrm{Al}$ foil ( 0.9 to 1.0 equiv, in small pieces), with slight warming at $40-50{ }^{\circ} \mathrm{C}$ in a water bath, after 18 to $20 \mathrm{~h}$ an addition reaction efficiently took place, and an aluminium alcoholate 3 was obtained that after aqueous work-up gave the homoallylic alcohol product 4 . We found that the optimal ratio of $\mathrm{In} / \mathrm{Al}$ was at about 1:10. Lower ratios can be used, but led to longer reaction times, or rquired higher reaction temperatures. If water was present during the reaction (or used as solvent), aluminium salts precipitated, and product $\mathbf{4}$ was obtained directly. We observed that, in the absence of In, the Barbier reaction failed.<smiles>O=CC1CCCCC1</smiles><smiles>[R]c1ccc(C=O)cc1[R]</smiles>

$1 \mathrm{a}$

1b: $\mathrm{R}^{1}=\mathrm{R}^{2}=\mathrm{H}$<smiles>O=Cc1c2ccccc2cc2ccccc12</smiles>

1e<smiles>COc1cc(OC)c(C=O)c(OC)c1</smiles>

1f<smiles>O=Cc1ccco1</smiles>

19

1c: $\mathrm{R}^{1}=\mathrm{H} ; \mathrm{R}^{2}=\mathrm{Cl}$

1d: $\mathrm{R}^{1}=\mathrm{Br} ; \mathrm{R}^{2}=\mathrm{H}$<smiles>[R]C1CCCCC1=O</smiles>

1h<smiles>COc1ccc2c(c1)CCCC2=O</smiles>

1n 1i: $\mathrm{R}^{3}=\mathrm{H}$

1j: $\mathrm{R}^{3}=\mathrm{CH}_{3}$<smiles>CC1(C)CC(=O)c2c(O)cccc2O1</smiles>

10 $\mathrm{CH}_{3}$<smiles>[R]C(=O)c1ccccc1</smiles>

1k: $\mathrm{R}^{4}=\mathrm{CH}_{3}$<smiles>O=c1c2ccccc2oc2ccccc12</smiles>

$1 \mathrm{~m}$<smiles>CC(=O)CCC=C(C)C</smiles>

$1 \mathrm{p}$<smiles>CC1=CCCC(C)(C)C1/C=C/C=O</smiles>

Scheme 2. Aldehydes and ketones used.

We observed that after about 30 min the originally shiny Al metal surface became opaque and as the reaction progressed, the originally clear or slightly colored solution turned greenish and then dark grey, probably due to the finely divided metal and product salts suspended, similar to our previous observation for the reaction with stoichiometric granular In. ${ }^{12 \mathrm{~b}}$ After around some $20 \mathrm{~h}$ of vigorous stirring, all the aldehydes and ketones $\mathbf{1}$ tested were consumed, as revealed by 
chromatographic analysis, and converted into the homoallyl alcohol $\mathbf{2}$ or, rather, its aluminium salt (Scheme 1). The results are summarized in Table 1 and all compounds used, are shown in Scheme 2.

Table 1. Indium catalyzed reaction of carbonyl compounds with allyl bromide

\begin{tabular}{cccc}
\hline Entry & Substrate & Product & Yield $(\%)^{\mathrm{a}}$ \\
\hline 1 & $\mathbf{1 a}$ & $\mathbf{4 a}$ & 70 \\
2 & $\mathbf{1 b}$ & $\mathbf{4 b}$ & 80 \\
3 & $\mathbf{1 c}$ & $\mathbf{4 c}$ & 60 \\
4 & $\mathbf{1 d}$ & $\mathbf{4 d}$ & 52 \\
5 & $\mathbf{1 e}$ & $\mathbf{4 e}$ & 80 \\
6 & $\mathbf{1 f}$ & $\mathbf{4 f}$ & 76 \\
7 & $\mathbf{1 g}$ & $\mathbf{4 g}$ & 72 \\
8 & $\mathbf{1 h}$ & $\mathbf{4 h}$ & 78 \\
9 & $\mathbf{1 i}$ & $\mathbf{4 i}$ & 87 \\
10 & $\mathbf{1 j}$ & $\mathbf{4 j}$ & 85 \\
11 & $\mathbf{1 k}$ & $\mathbf{4 k}$ & 87 \\
12 & $\mathbf{1 l}$ & $\mathbf{4 l}$ & 92 \\
13 & $\mathbf{1 m}$ & $\mathbf{4 m}$ & 90 \\
14 & $\mathbf{1 n}$ & $\mathbf{4 n}$ & 72 \\
15 & $\mathbf{1 0}$ & $\mathbf{4 o}$ & 80 \\
16 & $\mathbf{1 p}$ & $\mathbf{4 p}$ & 90 \\
17 & $\mathbf{1 q}$ & $\mathbf{4 q}$ & 75 \\
\hline
\end{tabular}

${ }^{a}$ Refers to isolated products, after column chromatography purification.

From the experimental data, it was possible to conclude that their reaction times and isolated yields were essentially similar either for aldehydes or ketones. Also, when yields were compared with those obtained for the stoichiometric reactions, most were basically the same, or a little lower in some cases. However, reaction times were much longer for the catalytic reaction.

\section{Conclusions}

In summary, we demonstrate that cheap household aluminium foil can give rise, in the presence of a catalytic amount of indium, to Barbier reactions of allyl bromide with aldehydes and ketones. This economical protocol can be easily scaled up to preparative amounts, and uses metals that are ecologically benign. 


\section{Experimental Section}

General. FTIR spectra were recorded on a Bruker Vector-22 spectrometer. ${ }^{1} \mathrm{H}$ spectra were recorded either at 400 or at $200 \mathrm{MHz}$, and ${ }^{13} \mathrm{C}$ spectra at 100 or at $50 \mathrm{MHz}$, on a Bruker DRX400 AVANCE, or a Bruker ACE-200 instrument, with $\mathrm{CDCl}_{3}$ as solvent and TMS as the internal standard. MS were recorded on a Shimadzu GCMS-QP5050A benchtop quadrupole mass spectrometer operating at $70 \mathrm{eV}$. HRMS data were obtained in a Thermo Finnigan MAT95XP spectrometer, either by FAB, CI, or EI. Elemental microanalyses for C, H and N were obtained using a Fisons CHNS-O microanalyzer. Allyl bromide, and all carbonyl compounds were purchased from Aldrich (USA), and were used without any further purification. All technicalgrade organic solvents, preparative "flash" Silica gel 60, and thin layer chromatography (TLC) plates type Silica gel $60 \mathrm{~F}_{254}$ on aluminium sheets, were purchased from Merck (Darmstadt, Germany). Analytical TLC plates were eluted with mixtures of hexane/EtOAc $(2: 1)$, and the compounds spots visualized with an UV lamp, iodine vapors, and/or sprayed with dilute sulfuric acid and heated. In all cases, the homogeneity of a compound in TLC and GC analysis, and its elemental microanalysis, was used as a proof of purity. All products described in this study were obtained as colorless oils, and were previously cited in the literature: $\mathbf{4 a},{ }^{12 b, 17 a-b} \mathbf{4 b},{ }^{12 b, 17 a-c}$ $\mathbf{4 c},{ }^{12 \mathrm{~b}, 17 \mathrm{c}, 17 \mathrm{~h}} \mathbf{4 d},{ }^{12 \mathrm{~b}, 17 \mathrm{~d}, 17 \mathrm{i}} \mathbf{4 e},{ }^{12 \mathrm{~b}, 17 \mathrm{e}} \mathbf{4 f},{ }^{12 \mathrm{~b}, 17 \mathrm{~g}} \mathbf{4 g},{ }^{12 \mathrm{~b}, 17 \mathrm{c}, 17 \mathrm{e}} \mathbf{4 h},{ }^{12 \mathrm{~b}, 17 \mathrm{c}} \mathbf{4 i},{ }^{12 \mathrm{~b}, 17 \mathrm{c}, 17 \mathrm{f}, 17 \mathrm{i}} \mathbf{4 j},{ }^{12 \mathrm{~b}, 17 \mathrm{f}} \mathbf{4 k},{ }^{12 \mathrm{~b}, 17 \mathrm{c}, 17 \mathrm{i}}$ $\mathbf{4 l},{ }^{12 \mathrm{~b}} \mathbf{4 m},{ }^{12 \mathrm{~b}} \mathbf{4 n},{ }^{2 \mathrm{~b}, 17 \mathrm{i}} \mathbf{4 o},{ }^{12 \mathrm{~b}} \mathbf{4} \mathbf{p},{ }^{12 \mathrm{~b}, 17 \mathrm{k}} \mathbf{4 q}{ }^{12 \mathrm{~b}}$

General synthetic procedure for the preparation of homoallyl alcohols 4 from carbonyl compounds 1, exemplified by 1-phenyl-3-butene-1-ol (4b):

Allyl bromide (484 mg, $4 \mathrm{mmol}$ ) and benzaldehyde (1b) (106 mg, $1 \mathrm{mmol}$ ) were dissolved in DMF ( $1 \mathrm{~mL})$, contained in a thick-wall reaction tube with threaded Teflon cap, and a stirring bar. Granular indium $(11.5 \mathrm{mg}, 0.1 \mathrm{mmol})$ and aluminium foil cut in small pieces $(27 \mathrm{mg}, 1 \mathrm{mmol})$ were added at once, the reaction tube was closed, and vigorously stirred into a prewarmed water bath with the temperature set at $40-50{ }^{\circ} \mathrm{C}$, and controlled by an immersion thermometer. The reaction progress was followed by TLC analysis, until total consumption of $\mathbf{2}$ was observed (overnight, 18-20 h). The resulting dark green suspension was diluted with EtOAc, poured into aqueous $\mathrm{N} / 10 \mathrm{HCl}(50 \mathrm{~mL})$, and extracted 3 times with the same volume of EtOAc. The combined extracts were washed 3 times with water, brine, dried $\left(\mathrm{Na}_{2} \mathrm{SO}_{4}\right)$ and evaporated in vacuo to yield crude homoallylic alcohol $\mathbf{4 b}$, which was purified by flash chromatography, to yield a colorless oil (118 mg, 80\%, see physical data below).

1-Cyclohexylbut-3-en-1-ol (4a): ${ }^{1} \mathrm{H}$ NMR (400 MHz): $\delta$ 5.90-5.70 (m, 1H), 5.20-5.10 (m, 2H), $3.43-3.31(\mathrm{~m}, 1 \mathrm{H}), 2.40-2.20(\mathrm{~m}, 1 \mathrm{H}), 2.18-2.05(\mathrm{~m}, 1 \mathrm{H}), 1.90-1.50(\mathrm{~m}, 6 \mathrm{H}), 1.45-0.90(\mathrm{~m}, 6 \mathrm{H})$ ppm. ${ }^{13} \mathrm{C}$ NMR (100 MHz): $\delta$ 137.7, 115.8, 73.3, 44.9, 40.1, 28.3, 27.6, 26.2 ppm. m/z $154\left(\mathrm{M}^{+}\right.$, $100 \%) ; 155(\mathrm{M}+1,11 \%) ; \mathrm{C}_{10} \mathrm{H}_{18} \mathrm{O}$ requires an exact mass of 154.14. Microanalysis: calcd. $\mathrm{C}$ 77.9, $\mathrm{H}$ 11.8; found C 77.5, H $12.0 \%$. 
1-Phenyl-3-butene-1-ol (4b): IR 3383, 3075, 1665, $1641 \mathrm{~cm}^{-1} .{ }^{1} \mathrm{H}$ NMR (200 MHz): $\delta$ 7.43-7.24 (m, 5H), 5.84-5.81 (m, 1H), 5.20-5.14 (m, 2H), 4.75 (t, $5.3 \mathrm{~Hz}, 1 \mathrm{H}), 2.57-2.49(\mathrm{~m}, 2 \mathrm{H}), 1.99$ (br $\mathrm{s}, 1 \mathrm{H}) .{ }^{13} \mathrm{C}$ NMR $(50 \mathrm{MHz}): \delta$ 143.8, 134.4, 128.3, 127.4, 125.7, 118.1, 73.2, 43.6. $\mathrm{m} / \mathrm{z} 148\left(\mathrm{M}^{+}\right.$, $100 \%), 149(\mathrm{M}+1,10 \%)$; HRMS: $148.0888 ; \mathrm{C}_{10} \mathrm{H}_{12} \mathrm{O}$ requires an exact mass of 148.0888 .

1-(3-Chlorophenyl)but-3-en-1-ol (4c): ${ }^{1} \mathrm{H}$ NMR (400 MHz): $\delta 7.55$ (d, $\left.2.5 \mathrm{~Hz}, 1 \mathrm{H}\right), 7.37-7.20$ $(\mathrm{m}, 3 \mathrm{H}), 5.78-5.62(\mathrm{~m}, 1 \mathrm{H}), 5.09-5.00(\mathrm{~m}, 2 \mathrm{H}), 4.72-4.51(\mathrm{~m}, 1 \mathrm{H}), 2.63-2.30(\mathrm{~m}, 2 \mathrm{H}) \mathrm{ppm} .{ }^{13} \mathrm{C}$ NMR (100 MHz): $\delta 145.5,134.1,132.8,129.3,127.3,125.6,123.3,118.5,72.1,43.5$ ppm. $\mathrm{m} / z$ $182\left(\mathrm{M}^{+}, 100 \%\right), 183(\mathrm{M}+1,11 \%), 184(\mathrm{M}+2,32 \%) ; \mathrm{C}_{10} \mathrm{H}_{11} \mathrm{ClO}$ requires an exact mass of 182.050. Microanalysis: calcd. C 65.8, H 6.1; found C 66.0, H 6.0\%.

1-(4-Bromophenyl)but-3-en-1-ol (4d): IR 3383, 3075, $1642 \mathrm{~cm}^{-1} .{ }^{1} \mathrm{H}$ NMR (200 MHz): $\delta 7.95$ (d, $8.3 \mathrm{~Hz}, 2 \mathrm{H}), 7.30$ (d, 8.3 Hz, 2H), 5.75 (ddt, 16.0, 8.0, 6.3 Hz, 1H), 5.15 (d, $16.0 \mathrm{~Hz}, 1 \mathrm{H})$, $5.13(\mathrm{~d}, 8.0 \mathrm{~Hz}, 1 \mathrm{H}), 4.72(\mathrm{t}, 6.2 \mathrm{~Hz}, 1 \mathrm{H}), 2.61-2.39(\mathrm{~m}, 2 \mathrm{H}) .{ }^{13} \mathrm{C} \mathrm{NMR}(50 \mathrm{MHz}): \delta 142.8$, 135.7, 132.0, 127.2, 122.8, 116.5, 75.8, 44.4 ppm. $\mathrm{m} / \mathrm{z}: 226\left(\mathrm{M}^{+}, 100 \%\right), 228(\mathrm{M}+2,97 \%)$; HRMS: 225.9998; $\mathrm{C}_{10} \mathrm{H}_{11} \mathrm{BrO}$ requires an exact mass of 225.999. Microanalysis: calcd. C 52.9, H 4.9; found C 53.0, H 4.9\%.

1-(Anthracen-9-yl)but-3-en-1-ol (4e): ${ }^{1} \mathrm{H}$ NMR (400 MHz): $\delta 8.20$ (s, 1H), 8.00-7.90 (m, 4H), 7.40-7.35 (m, 4H), 5.82 (ddt, 15.6, 8.4 and 6.1 Hz, 1H), 5.10 (d, $15.7 \mathrm{~Hz}, 1 \mathrm{H}), 5.05$ (d, $8.4 \mathrm{~Hz}$, 1H), 4.55 (t, $6.0 \mathrm{~Hz}, 1 \mathrm{H}), 2.58-2.40(\mathrm{~m}, 2 \mathrm{H}) .{ }^{13} \mathrm{C}$ NMR (100 MHz): $\delta 137.2,134.4,131.3,129.0$, 126.0, 125.8, 125.6, 116.2, 72.8, 43.1 ppm. $m / z 248\left(\mathrm{M}^{+}, 100 \%\right)$; HRMS: 248.3192; $\mathrm{C}_{18} \mathrm{H}_{16} \mathrm{O}$ requires an exact mass of 248.3190. Microanalysis: calcd. C 87.1, H 6.5; found C 87.0, H 6.5\%.

1-(2,4,6-Trimethoxyphenyl)but-3-en-1-ol (4f): ${ }^{1} \mathrm{H}$ NMR (400 MHz): $\delta 6.25$ (s, 2H), 5.87 (ddt, 15.8, 8.1 and $6.3 \mathrm{~Hz}, 1 \mathrm{H}), 5.07$ (d, $15.6 \mathrm{~Hz}, 1 \mathrm{H}), 5.03(\mathrm{~d}, 8.1 \mathrm{~Hz}, 1 \mathrm{H}), 4.65$ (t, $6.4 \mathrm{~Hz}, 1 \mathrm{H}), 3.81$ $(\mathrm{s}, 6 \mathrm{H}), 3.76(\mathrm{~s}, 3 \mathrm{H}), 3.65$ (br s, 1H), 2.60-2.37 (m, 2H). ${ }^{13} \mathrm{C}$ NMR (100 MHz): $\delta$ 162.2, 155.9, 133.5, 116.4, 102.0, 93.0, 65.0, 55.4, 55.1, 43.0 ppm. m/z $238\left(\mathrm{M}^{+}, 100 \%\right), 239$ (M+1, 14\%); HRMS: 238.1205; $\mathrm{C}_{13} \mathrm{H}_{18} \mathrm{O}_{4}$ requires an exact mass of 238.1205. Microanalysis: calcd. C 65.5, $\mathrm{H}$ 7.6; found C 65.4, $\mathrm{H} \mathrm{7.6 \% .}$

1-(2-Furfuryl)but-3-en-l-ol (4g): IR 3385, 1669, $1642 \mathrm{~cm}^{-1} .{ }^{1} \mathrm{H}$ NMR (400 MHz) $\delta 7.70$ (d, 2.0 $\mathrm{Hz}, 1 \mathrm{H}), 6.43$ (dd, 2.0, 3.0 Hz, 1H), 6.40 (d, 3.0 Hz, 1H), 5.81 (ddd, 16.0, 8.4, 6.2 Hz, 1H), 5.05 $(\mathrm{d}, 16.0 \mathrm{~Hz}, 1 \mathrm{H}), 5.01(\mathrm{~d}, 8.4 \mathrm{~Hz}, 1 \mathrm{H}), 4.56(\mathrm{t}, 6.0 \mathrm{~Hz}, 1 \mathrm{H}), 3.50$ (br s, 1H), 2.65-2.53 (m, 1H), 2.49-2.46 (m, 1H). ${ }^{13} \mathrm{C}$ NMR (50 MHz): $\delta$ 155.1, 142.6, 131.7, 116.5, 110.1, 108.7, 72.0, 41.6 ppm. Microanalysis: calcd. C 69.5, H 7.3; found C 69.8, H 7.3\%.

6,10-Dimethylundeca-l,5,9-trien-4-ol (4h): IR 3356, 1669, $1641 \mathrm{~cm}^{-1} .{ }^{1} \mathrm{H}$ NMR (200 MHz) $\delta$ 5.86-5.80 (m, 1H), 5.40-5.10 (m, 4H), 4.18-4.05 (m, 1H), 2.40-1.90 (m, 6H), $1.65(\mathrm{~s}, 6 \mathrm{H}), 1.59$ (s, 3H), 1.23 (br s, 1H). ${ }^{13} \mathrm{C}$ NMR (50 M Hz): $\delta$ 140.1, 139.7, 131.4, 127.8, 124.5, 119.5, 68.3, 44.6, 34.0, 27.5, 24.6, 22.7, 18.7 ppm. $\mathrm{m} / \mathrm{z} 194\left(\mathrm{M}^{+}, 100 \%\right), 195(\mathrm{M}+1,14 \%) ; \mathrm{C}_{13} \mathrm{H}_{22} \mathrm{O}$ requires an exact mass of 194.1671. Microanalysis: calcd. C 80.4, H 11.4; found C 80.5, H 11.5\%.

1-Allylcyclohexanol (4i): ${ }^{1} \mathrm{H}$ NMR (400 MHz): $\delta 5.82$ (ddt, 16.2, 8.7, 6.5 Hz, 1H), 5.07-5.02 (m, 2H), 4.65 (br s, 1H), 2.30-2.10 (m, 2H), 1.70-1.55 (m, 4H), 1.50-1.40 (m, 6H) ppm. ${ }^{13} \mathrm{C} \mathrm{NMR}$ (100 MHz): $\delta$ 134.0, 118.4, 72.1, 47.4, 42.0, 26.4, 22.0 ppm. $m / z$ (CI) $141\left(\mathrm{MH}^{+}, 100 \%\right)$. 
$\left(\mathrm{C}_{9} \mathrm{H}_{16} \mathrm{O}\right.$ requires an exact mass of 140.1201). Microanalysis: calcd. C 77.1, H 11.5; found $\mathrm{C}$ 77.0, $\mathrm{H} 11.3 \%$.

1-Allyl-2-methylcyclohexanol (4j): IR 3483, 3075, 2931, $1639 \mathrm{~cm}^{-1} .{ }^{1} \mathrm{H}$ NMR (200 MHz): $\delta$ $5.78(\mathrm{ddt}, 16.1,8.5,6.2 \mathrm{~Hz}, 1 \mathrm{H}), 5.07(\mathrm{dd}, 16.0,2.0 \mathrm{~Hz}, 1 \mathrm{H}), 5.00(\mathrm{dd}, 8.6,2.0 \mathrm{~Hz}, 1 \mathrm{H}), 3.60(\mathrm{br}$ s, $1 \mathrm{H}), 2.20-1.90(\mathrm{~m}, 2 \mathrm{H}), 1.68-1.50(\mathrm{~m}, 4 \mathrm{H}), 1.48-1.25(\mathrm{~m}, 5 \mathrm{H}), 1.00(\mathrm{~d}, 6.7 \mathrm{~Hz}, 3 \mathrm{H}) \mathrm{ppm} .{ }^{13} \mathrm{C}$ NMR (50 MHz): $\delta$ 132.7, 116.3, 88.2, 42.4, 41.3, 39.4, 31.4, 26.1, 23.4, 17.6 ppm. $\mathrm{m} / z$ (CI) 155 $\left(\mathrm{MH}^{+}, 100 \%\right) ; \mathrm{C}_{10} \mathrm{H}_{18} \mathrm{O}$ requires an exact mass of 154.1358 . Microanalysis: calcd. $\mathrm{C} 77.9, \mathrm{H}$ 11.8; found $\mathrm{C} 80.1, \mathrm{H} 11.6 \%$.

2-Phenylpent-4-en-2-ol (4k): ${ }^{1} \mathrm{H}$ NMR (400 MHz): $\delta$ 7.60-7.35 (m, 5H), 5.76 (ddt, 15.7, 8.7, 6.5 $\mathrm{Hz}, 1 \mathrm{H}), 5.12$ (dd, 15.7, $2.5 \mathrm{~Hz}, 1 \mathrm{H}), 5.02(\mathrm{dd}, 8.6,2.5 \mathrm{~Hz}, 1 \mathrm{H}), 2.55-2.35(\mathrm{~m}, 2 \mathrm{H}), 1.35(\mathrm{~s}, 3 \mathrm{H})$ ppm. ${ }^{13} \mathrm{C}$ NMR (100 MHz): $\delta 148.0,135.8,129.0,127.5,126.2,81.2,57.7,33.0$ ppm. $m / z 162$ $\left(\mathrm{M}^{+}, 100 \%\right) ; \mathrm{C}_{11} \mathrm{H}_{14} \mathrm{O}$ requires an exact mass of 162.1045 . Microanalysis: calcd. C 81.4, H 8.7; found $\mathrm{C} 81.1, \mathrm{H} 8.4 \%$.

1,1-Diphenylbut-3-en-1-ol (4I): ${ }^{1} \mathrm{H}$ NMR (400 MHz): $\delta$ 7.60-7.30 (m, 10H), 5.80-5.60 (m, 1H), 5.12 (br d, $15.8 \mathrm{~Hz}, 1 \mathrm{H}), 5.02$ (br d, $8.9 \mathrm{~Hz}, 1 \mathrm{H}), 2.73$ (d, $6.7 \mathrm{~Hz}, 2 \mathrm{H}) \mathrm{ppm} .{ }^{13} \mathrm{C}$ NMR $(100$ MHz): $\delta 144.3,137.0,129.4,128.2,125.8,118.7,85.3,62.7$ ppm. $\mathrm{m} / z 224\left(\mathrm{M}^{+}, 100 \%\right), 225$ $(\mathrm{M}+1,17 \%), 226(\mathrm{M}+2,2 \%) ; \mathrm{C}_{16} \mathrm{H}_{16} \mathrm{O}$ requires an exact mass of 224.1201. Microanalysis: calcd. C 85.7, H 7.2; found C 85.4, H 7.3\%.

9-Allyl-9H-xanthen-9-ol (4m): ${ }^{1} \mathrm{H}$ NMR (400 MHz): $\delta$ 7.35-7.10 (m, 8H), 5.80-5.60 (m, 1H), 5.10-5.02 (m, 2H), 2.67 (s, 2H) ppm. ${ }^{13} \mathrm{C}$ NMR (100 MHz): $\delta$ 155.6, 132.1, 129.7, 126.2, 122.4, 118.7, 79.6, 48.0 ppm. $m / z 238\left(\mathrm{M}^{+}, 100 \%\right), 239(\mathrm{M}+1,17 \%), 240(\mathrm{M}+2,1 \%) ; \mathrm{C}_{16} \mathrm{H}_{14} \mathrm{O}_{2}$ requires an exact mass of 238.0994. Microanalysis: calcd. C 80.7, H 5.9; found C 80.9, H 5.4\%.

1-Allyl-6-methoxy-1,2,3,4-tetrahydronaphthalen-1-ol (4n): ${ }^{1} \mathrm{H}$ NMR (400 MHz): $\delta 6.80$ (d, $9.0 \mathrm{~Hz}, 1 \mathrm{H}), 6.78$ (d, $3.4 \mathrm{~Hz}, 1 \mathrm{H}), 6.44$ (dd, 9.0, $3.4 \mathrm{~Hz}, 1 \mathrm{H}), 5.84$ (ddt, 16.0, 8.7, 6.7 Hz, 1H), 5.08-5.02 (m, 2H), $3.78(\mathrm{~s}, 3 \mathrm{H}), 2.74-2.37(\mathrm{~m}, 4 \mathrm{H}), 1.87-1.60(\mathrm{~m}, 4 \mathrm{H}) \mathrm{ppm} .{ }^{13} \mathrm{C}$ NMR $(100$ MHz): $\delta$ 157.1, 140.9, 135.6, 130.2, 129.1, 127.5, 113.7, 111.4, 78.1, 55.6, 54.6, 35.6, 31.8, 21.2 ppm. $m / z 218\left(\mathrm{M}^{+}, 100 \%\right), 219(\mathrm{M}+1,15), 220(\mathrm{M}+2,1) ; \mathrm{C}_{14} \mathrm{H}_{18} \mathrm{O}_{2}$ requires an exact mass of 218.1307. Microanalysis: calcd. C 77.0, H 8.3; found C 76.9, H 8.0\%.

4-Allyl-2,2-dimethylchroman-4,5-diol (4o): IR 3319, 3076, 2977, 1709, 1665, $1639 \mathrm{~cm}^{-1} .{ }^{1} \mathrm{H}$ NMR (200 MHz): $\delta 6.80(\mathrm{~d}, 9.0 \mathrm{~Hz}, 1 \mathrm{H}), 6.78(\mathrm{~d}, 3.4 \mathrm{~Hz}, 1 \mathrm{H}), 6.44(\mathrm{dd}, 9.0,3.4 \mathrm{~Hz}, 1 \mathrm{H}), 5.84$ (ddt, 16.0, 8.7, $6.7 \mathrm{~Hz}, 1 \mathrm{H}), 5.08-5.02(\mathrm{~m}, 2 \mathrm{H}), 3.78(\mathrm{~s}, 3 \mathrm{H}), 2.74-2.37(\mathrm{~m}, 4 \mathrm{H}), 1.87-1.60(\mathrm{~m}$, 4H) ppm. ${ }^{13} \mathrm{C}$ NMR $(50 \mathrm{MHz}): \delta 157.1,140.9,130.2,129.1,127.5,113.7,111.4,78.1,55.6$, 35.6, 31.8, $21.2 \mathrm{ppm} . \mathrm{m} / \mathrm{z} 234\left(\mathrm{M}^{+}, 100 \%\right) ; \mathrm{C}_{14} \mathrm{H}_{18} \mathrm{O}_{2}$ requires an exact mass of 234.1256 . Microanalysis: calcd. C 71.8, $\mathrm{H} 7.7$; found C 72.0, $\mathrm{H} 8.0 \%$.

4,8-Dimethylnona-1,7-dien-4-ol (4p): ${ }^{1} \mathrm{H}$ NMR (400 MHz): $\delta$ 5.85-5.80 (m, 1H), 5.25-5.15 (m, 1H), 5.05-4.94 (m, 2H), 3.78 (br s, 1H), 2.21-2.18 (m, 1H), 2.00-1.80 (m, 3H), 1.72 (s, 3H), 1.65 (s, 3H), 1.50-1.44 (m, 2H), 1.29 (s, 3H) ppm. ${ }^{13} \mathrm{C}$ NMR (100 MHz): $\delta 134.1,132.0,125.2,118.1$, 75.4, 50.6, 43.6, 31.4, 24.7, 22.0, 18.7 ppm. $\mathrm{m} / \mathrm{z} 168\left(\mathrm{M}^{+}, 100 \%\right), 169(\mathrm{M}+1,12) ; \mathrm{C}_{11} \mathrm{H}_{20} \mathrm{O}$ requires an exact mass of 168.1514. Microanalysis: calcd. C 78.5, $\mathrm{H} 12.0$; found $\mathrm{C} 78.3, \mathrm{H}$ $12.1 \%$. 
1-(2,6,6-Trimethylcyclohex-2-en-1-yl)hexa-1,5-dien-3-ol (4q): ${ }^{1} \mathrm{H}$ NMR (400 MHz): $\delta$ 5.85$5.52(\mathrm{~m}, 3 \mathrm{H}), 5.42-5.32(\mathrm{~m}, 1 \mathrm{H}), 5.10-5.00(\mathrm{~m}, 2 \mathrm{H}), 4.02-3.90(\mathrm{~m}, 1 \mathrm{H}), 2.62-2.50(\mathrm{~m}, 2 \mathrm{H}), 2.28-$ $2.25(\mathrm{~m}, 1 \mathrm{H}), 2.05-1.90(\mathrm{~m}, 3 \mathrm{H}), 1.78(\mathrm{~s}, 3 \mathrm{H}), 1.70-1.65(\mathrm{~m}, 1 \mathrm{H}), 1.50-1.46(\mathrm{~m}, 1 \mathrm{H}), 1.02(\mathrm{~s}$, $3 \mathrm{H}), 0.90(\mathrm{~s}, 3 \mathrm{H}) \mathrm{ppm} .{ }^{13} \mathrm{C} \mathrm{NMR}(100 \mathrm{MHz}): \delta$ 135.0, 131.3, 128.8, 122.4, 120.3, 119.5, 73.2, 57.2, 43.5, 33.1, 30.9, 28.6, 27.2, 22.5, 21.5 ppm. $\mathrm{m} / \mathrm{z} 220\left(\mathrm{M}^{+}, 100 \%\right), 221(\mathrm{M}+1,16), 222$ $(\mathrm{M}+2,1) ; \mathrm{C}_{15} \mathrm{H}_{24} \mathrm{O}$ requires an exact mass of 220.1827. Microanalysis: calcd. C 81.8, H 11.0; found $\mathrm{C} 82.0,11.2 \%$.

\section{Acknowledgements}

The authors are grateful to "Pontificia Universidad Católica de Chile" (PUC) (Vicerrectoría Adjunta de Investigación y Doctorado, Proyecto Límite 04/2009), and "Facultad de Química PUC" (Proyecto interno), for financial support.

\section{References}

1. For reviews: (a) Courtois, G.; Miginiac, L. J. Organomet. Chem. 1974, 69, 1. (b) Biellmann, J. F.; Ducep, J. B. Org. React. 1983, 27, 1. (c) Roush, R. W. in Comprehensive Organic Synthesis, Trost, B. M.; Fleming, I.; Heathcock, C. H. Eds; Pergamon: Oxford, 1991; vol. 2, p. 1.

2. (a) Pearce, P. J.; Richard, D. H.; Scilly, N. F. J. Chem. Soc., Perkin Trans. 1 1971, 1655. (b) Luche, J. L.; Damiano, J. C. J. Am. Chem. Soc. 1980, 102, 7927.

3. Blomberg, C.; Hartog, F. A. Synthesis 1977, 18.

4. (a) Hiyama, T.; Obayashi, M.; Nakamura, A. Organometallics 1982, 1, 1249. (b) Hiyama, T.; Sawahata, M.; Kusano, Y. Chem. Lett. 1985, 611.

5. (a) Hiyama, T.; Sawahata, M.; Obayashi, M. Chem. Lett. 1983, 1237. (b) Boldrini, G. P.; Savoia, D.; Tagliavini, E.; Trombin, C.; Umani-Ronchi, A. J. Org. Chem. 1983, 48, 4108. (c) Perier, C.; Luche, J. L. J. Org. Chem. 1982, 50, 910. (d) Peter, C.; Einhorn, J.; Luche, J. L. Tetrahedron Lett. 1985, 26, 1449.

6. (a) Mukaiyama, T.; Warada, T.; Shoda, S. Chem. Lett. 1980, 1207. (b) Nokami, J.; Otera, J.; Sudo, T.; Okawara, R. Organometallics 1983, 2, 191.

7. Tanaka, H.; Yamashita, S.; Hamatani, T.; Ikemoto, Y.; Torii, S. Chem. Lett. 1986, 1611.

8. (a) Wada, M.; Akiba, K. Tetrahedron Lett. 1985, 26, 4211. (b) Wada, M.; Ohki, H.; Akiba, K. Tetrahedron Lett. 1986, 27, 4771. (c) Wada, M.; Ohki, H.; Akiba, K. J. Chem. Soc., Chem. Commun. 1987, 708.

9. Imamoto, T.; Kusumoto, T.; Tawarayama, Y.; Sugiura, Y.; Mita, T. J. Org. Chem. 1984, 49, 3904. 
10. (a) Okude, Y.; Hirano, S.; Hiyama, T.; Nozaki, H. J. Am. Chem. Soc. 1977, 99, 3179. (b) Buse, C. T.; Heathcock, C. H. Tetrahedron Lett. 1978, 19, 1685. (c) Souppe, J.; Namy, J. L.; Kagan, B. H. Tetrahedron Lett. 1982, 23, 3497. (d) Butugan, Y.; Ito, H.; Araki, S. Tetrahedron Lett. 1987, 28, 3707.

11. For in depth reviews: (a) Nair, V.; Ros, S.; Jayan, C. N.; Pillai, D. S. Tetrahedron 2004, 60, 1959. (b) Poddlech, J.; Maier, T. C. Synthesis 2003, 633; (c) Cintas, P. Synlett 1995, 1087.

12. (a) Lin, C. J. Tetrahedron 1996, 52, 5643. (b) Preite, M. D.; Pérez-Carvajal, A. Synlett 2006, 3337.

13. (a) Downs, A. J. Chemistry of Aluminium, Gallium, Indium and Thallium, First Edn.; Chapman \& Hall: Glasgow, 1993; pp 89 and 106. (b) For a recent review: Roy, U. K.; Roy, S. Chem. Rev. 2010, 110, 2472, and references cited.

14. (a) Araki, S.; Jin, S. J.; Idou, Y.; Butsugan, Y. Bull. Chem. Soc. Jpn. 1992, 65, 1736. (b) Steurer, S.; Podlech, J. Adv. Synth. Catal. 2001, 343, 251. (c) Auge, J.; Lubin-Germain, N.; Woaye, A. T. Tetrahedron Lett. 1999, 40, 9245. (d) Auge, J.; Lubin-Germain, N.; Marque, S.; Seghrouchni, L. J. Organomet. Chem. 2003, 679, 79. (e) Lombardo, M.; Morganti, S.; Trombini, C. J. Org. Chem. 2003, 68, 997. (f) Lombardo, M.; Girotti, R.; Morganti, S.; Trombini, C. Org. Lett. 2001, 3, 2981.

15. Shen, K. H.; Yao, C. F. J. Org. Chem. 2006, 71, 3980.

16. We found that any form of Al foil can be used, but best results were obtained with the cheapest, thinner foils, that we cut into narrow stripes 1-2 mm wide, and then again in the across direction using scissors, to get as small as possible "flakes" of Al metal.

17. (a) Chen, W.; Liu, Y.; Chen. Z. Eur. J. Org. Chem. 2005, 1665. (b) Solin, N.; Kjellgren, J.; Szabò, K. J. J. Am. Chem. Soc. 2004, 126, 7026, and references cited therein. (c) Huang, Y.; Liao, Y. J. Org. Chem. 1991, 56, 1381. (d) Doucet, H.; Santelli, M. Tetrahedron: Asymm. 2000, 11, 4163, and references cited therein. (e) Zhang, Y.; Jia, X.; Wang, J.-X. Eur. J. Org. Chem. 2009, 2983, and references cited therein. (f) Takahara, J. P.; Masuyama, Y.; Kurusu, Y. J. Am. Chem. Soc. 1992, 114, 2577, and references cited therein. (g) Lal, K.; Ghosh, S.; Salomon, R. G. J. Org. Chem. 1987, 52, 1072, and references cited therein. (h) Haddad, T. D.; Hirayama, L. C.; Singaram, B. J. Org. Chem. 2010, 75, 642, and references cited therein. (i) Yamaguchi, M.; Morita, N.; Schneider, U.; Kobayashi, S. Adv. Synth. Catal. 2010, 352, 1461. (j) Cahiez, G.; Chavant, P.-Y. Tetrahedron Lett. 1989, 52, 7373, and references cited therein. (k) Takahashi, H.; Kato, N.; Iwashima, M.; Iguchi, K. Chem. Lett. 1999, 11, 1181. 